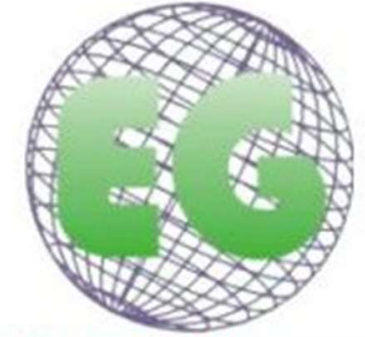

ISSN 1695-6141 N'57

\title{
Evaluación del riesgo de diabetes mellitus tipo 2 en pacientes afectados con tuberculosis
}

Risk assessment of type 2 diabetes mellitus in patients affected by tuberculosis

\author{
Jonathan R. Velasco ${ }^{1}$ \\ Jesús A. Cunalema ${ }^{1}$ \\ Ivana M. Poveda ${ }^{1}$ \\ Melba R. Eguiguren ${ }^{1}$ \\ Milka M. Domínguez ${ }^{2}$
}

\begin{abstract}
${ }^{1}$ Licenciado en Enfermería. Universidad de Guayaquil. Ecuador. jonathan.velascoy@ug.edu.ec
${ }^{2}$ Magister en Gerencia de Servicios de Salud. Docente de la Carrera de Enfermería, Facultad de Ciencias Médicas de la Universidad de Guayaquil. Ecuador.
\end{abstract}

http://dx.doi.org/10.6018/eglobal.19.1.357981

Recibido: 11/01/2019

Aceptado: 20/02/2019

\section{RESUMEN}

Introducción: La diabetes mellitus y la tuberculosis son problemas de salud emergentes a nivel mundial, su incidencia anual cada vez es mayor pese a todos los esfuerzos que se hacen por mitigarlas y su asociación podría ser un futuro problema de salud a enfrentar. A través de los años, diferentes investigaciones han demostrado que la Diabetes mellitus incrementa el riesgo de contraer tuberculosis entre 2 y 4 veces, es decir, es un factor predisponente pero no existen investigaciones que definan este riesgo de forma inversa.

Objetivo: Determinar el riesgo de desarrollar DM2 en pacientes afectados con tuberculosis.

Material y método: Se realizó un estudio cuantitativo, descriptivo y transversal, la población fueron 60 usuarios que asisten a tomar el tratamiento antifímico en 4 unidades operativas del Distrito de Salud 09D04, zona 8 a quienes se les aplico el test de Findrisc.

Resultados: El género masculino presenta un mayor riesgo de desarrollar DM2 junto con el grupo etario de $<45$ años y 45 - 54 años, de forma general el $48 \%$ tienen un nivel de riesgo bajo.

Conclusión: La población de pacientes afectados con tuberculosis, aunque presentan niveles de riesgo bajo, este se encuentra presente y es necesario tomar medidas para mitigar este riesgo y prevenir comorbilidades.

Palabras clave: riesgo, tuberculosis, diabetes mellitus tipo 2, pacientes.

\begin{abstract}
Introduction: The diabetes mellitus and tuberculosis are emergent health problems around the world its impact has been growing and growing every year even though health professionals do their best effort to eradicate them and these diseases mutations could be a real health problem to fight against in the future. Through the years, different researches have demostrate that Diabetes mellitus increase the risk of getting sick of tuberculosis 2 and 4 times more frequently, it means it is a predisposing factor, but there are not researches that define this risk in a backward effect.
\end{abstract}

Target: Establish risks of developing Diabetes Mellitus type 2 in patients affected by tuberculosis. 
Material and Method: A Quantitative, descriptive and transversal study was done, the statistical population was 60 users that take antifimic treatment in 4 Health Centers of District 09D04, zone 8. These users were tested through Findrisc Test.

Results: Male patients have more risk of developing Diabetes Mellitus type 2 at the age below 45 years old and between 45 and 54 years old, in general form $48 \%$ of patients have a low level risk.

Conclusion: The population of patients affected by tuberculosis has a low risk level and it is necessary take actions to mitigate this risk and prevent comorbidity.

Keywords: Risk, tuberculosis, diabetes mellitus Type 2, patients.

\section{INTRODUCCIÓN}

La tuberculosis es una de las 10 principales causas de mortalidad en el mundo. En 2016, 10,4 millones de personas enfermaron de tuberculosis y 1,7 millones murieron por esta enfermedad (entre ellos, 0,4 millones de personas con $\mathrm{VIH}$ ). Más del $95 \%$ de las muertes por tuberculosis se producen en países de ingresos bajos y medianos. La tuberculosis es una de las causas principales de defunción en las personas VIHpositivas: en 2016 , el $40 \%$ de las muertes asociadas al VIH se debieron a la tuberculosis. Se estima que entre 2000 y 2016 se salvaron 53 millones de vidas gracias a la dispensación de servicios de diagnóstico y tratamiento contra la tuberculosis.(1,2). Según la cohorte de 2014 , el tratamiento exitoso en el país para los casos nuevos y recaídas fue del $76,87 \%$. La tasa de mortalidad reportada por el Instituto Nacional de Estadística y Censos (INEC) es de 2,59/100 mil habitantes en 2014. (3)

En la actualidad el abordaje del sistema de salud del país está encaminado a brindar atención de salud prioritaria a los grupos vulnerables y/o con factores de riesgo acorde a los ciclos de vida; dentro de estos grupos están los pacientes con enfermedades crónicas transmisibles como la tuberculosis y no transmisibles como la diabetes, de las cuales desde el siglo XI Avicena documentó la asociación entre Tuberculosis (TB) y Diabetes Mellitus (DM) y junto con el incremento mundial de la DM en las últimas décadas esto ha repercutido en el aumento de los casos de TB-DM con importantes implicaciones clínicas, sociales y económicas ${ }^{(4)}$.

La diabetes mellitus (DM) afecta a 55 millones de personas en las Américas y se espera que su prevalencia aumente a 83 millones para $2030{ }^{(5)}$, en la clasificación de DM, tenemos la tipo 1 y 2; la DM tipo 2 (DM2) está asociada a la deficiencia en la secreción y/o acción de la insulina producida por las células beta del páncreas, siendo esta progresiva y crónica, originando una respuesta inmune innata que es la causante de varios daños a nivel tisular (4). Varios estudios han sugerido que la diabetes mellitus (DM) aumenta el riesgo de padecer tuberculosis activa (TB). La prevalencia creciente de $\mathrm{DM}$ en áreas endémicas de TB puede afectar negativamente el control de la TB ${ }^{(6)}$, estos estudios señalan que la tuberculosis podría inducir intolerancia a la glucosa y empeorar el control glucémico en personas con diabetes. En el contexto de la creciente superposición de poblaciones en riesgo de ambas enfermedades, la combinación de tuberculosis y diabetes mellitus representa una amenaza para la salud ya que podría afectar negativamente los esfuerzos mundiales de control de la tuberculosis ${ }^{(7,8)}$. La DM incrementa el riesgo de contraer TB entre 2 y 4 veces ${ }^{(9)}$, dependiendo de la población ${ }^{(10)}$ y está presente en un estimado del $15 \%$ de los casos de TB con un riesgo cuatro veces mayor de falla del tratamiento y un riesgo de muerte seis veces mayor. ${ }^{(11,12)}$

En la segunda edición 2018 de la Guía de Práctica Clínica (13) para la prevención, diagnóstico, tratamiento y control de la tuberculosis en el Ecuador, se recomienda 
evaluar el riesgo de DM a todo afectado por TB mediante el cuestionario FINDRISC(14) y si amerita realizar tamizaje por glucemia. La puntuación de riesgo de diabetes finlandés (FINDRISC) es uno de los instrumentos más utilizados a nivel mundial para evaluar el riesgo de DM2, consta de sólo ocho variables asociadas con parámetros antropométricos y factores de estilo de vida ${ }^{(15)}$.

Debido a la relación bidireccional de morbilidad que existe entre la DM y TB y con el objetivo de poder evaluar el riesgo de padecer DM2 se formuló la siguiente pregunta de investigación: ¿Cuál es el riesgo de desarrollar Diabetes Mellitus Tipo 2 en los pacientes afectados de tuberculosis? Conocer este riesgo va a permitir que los profesionales de la salud encargados del manejo de pacientes con TB intervengan de forma oportuna en la mitigación de estos riesgos y así poder brindar una mejor adherencia terapéutica al tratamiento antifímico.

\section{MATERIAL Y MÉTODO}

Hasta la fecha se han desarrollado e introducido varios métodos para cuantificar el riesgo de desarrollar DM2, sin embargo, en la actualidad el test de FINDRISC es el más aceptado por la comunidad científica. Para la aplicación de este test se realizó un estudio de tipo descriptivo, transversal, no experimental con un enfoque cuantitativo; para la recogida de datos se encuestó mediante el test de FINDRISC a una población de 60 pacientes que asisten a las diferentes unidades operativas del Distrito 09D04, Zona 8 a tomar el tratamiento antifímico; las variables de la investigación fueron el sexo, edad y riesgo de desarrollar DM2 en los pacientes; los criterios de inclusión que se utilizaron fueron: pacientes diagnosticados con TB en cualquiera de sus formas y que asistieron a tomar el medicamento el día de la encuesta; los criterios de exclusión fueron: pacientes con diagnóstico de DM de cualquier tipo, pacientes que asisten por tratamiento preventivo de isoniazida (TPI), pacientes que asisten a tomar el tratamiento por ser poblaciones de riesgo $(\mathrm{VIH})$. Para evitar sesgos en la información la encuesta fue anónima.

\section{Aspectos éticos}

Los individuos que participaron en el estudio firmaron un consentimiento informado en el cual se expuso el respeto al buen nombre, la salud, la confidencialidad, la intimidad y la dignidad, asimismo se les explicó todos los detalles concernientes al estudio y que en todo momento se iba a conservar el anonimato.

\section{RESULTADOS}

Una vez aplicado el test de Findrisc simultáneamente a los pacientes que asisten a tomar el tratamiento antifímico en las diferentes unidades operativas del Distrito de Salud 09D04, se logró cuantificar el riesgo de padecer DM2, para un mejor análisis estadístico, los datos se presentaron en tablas de asociación de variables y gráficos.

En la tabla 1 se presenta la asociación entre la variable sexo y el nivel de riesgo de padecer DM2 en los pacientes afectados con tuberculosis, en la tabla se puede observar predominancia del sexo masculino con un $71,7 \%$ que representa a 43 pacientes y un $17 \%$ de mujeres; en cuanto al nivel de riesgo tenemos que para ambos sexos el riesgo predominante se ubica en el nivel bajo con $35 \%$ y $13,3 \%$ respectivamente para hombres y mujeres, seguido por el nivel ligeramente elevado 
con un $26,7 \%$ y $6,7 \%$ respectivamente, para el rango de riesgo muy alto, el sexo masculino es el único que presenta datos con el 1,7\% que representa a 1 paciente.

Tabla 1. Relación entre la variable sexo y el nivel de riesgo de DM2 en los pacientes afectados de tuberculosis.

\begin{tabular}{cccccccccccccc}
\hline $\begin{array}{c}\text { Nivel de } \\
\text { riesgo de } \\
\text { DM2 }\end{array}$ & Bajo & \multicolumn{1}{c}{$\begin{array}{c}\text { Modera } \\
\text { do }\end{array}$} & $\begin{array}{c}\text { Ligeramente } \\
\text { elevado }\end{array}$ & Alto & $\begin{array}{c}\text { Muy } \\
\text { alto }\end{array}$ & $\begin{array}{c}\text { Total } \\
\mathbf{H}-\mathbf{M}\end{array}$ \\
\cline { 2 - 13 } & $N^{\circ}$ & $\%$ & $N^{\circ}$ & $\%$ & $N^{\circ}$ & $\%$ & $N^{\circ}$ & $\%$ & $N^{\circ}$ & $\%$ & $N^{\circ}$ & $\%$ \\
$\begin{array}{c}\text { Sexo } \\
\text { Masculino }\end{array}$ & 21 & 35 & 3 & 5 & 16 & 26,7 & 2 & 3,3 & 1 & 1,7 & 43 & 71,7 \\
Femenino & 8 & 13,3 & 3 & 5 & 3 & 5 & 3 & 5 & 0 & 0 & 17 & 28,3 \\
\hline
\end{tabular}

En la tabla 2 se analizó las edades de los pacientes y su relación con el riesgo de desarrollar DM2, para una mejor representación se los agrupó por grupos etarios según lo definido en el test de Findrisc. Respecto a la edad, el rango predominante es el de $<45$ años con un 66,7\%, seguido por el rango de 45 - 54 años con el 16,7\%, luego los rangos de 55 - 64 años y > 64 años representan el 8,4\% cada uno del total de participantes; con respecto a la relación entre edad y el estado del riesgo, los resultados más importantes por cada nivel reflejan que solo 1,7\% correspondiente a 1 paciente $>64$ años tiene un riesgo muy alto de desarrollar DM2; 10 pacientes del rango $<45$ años que representan el $16,7 \%$ tiene un riesgo ligeramente elevado; el $8,3 \%$ correspondiente a 5 paciente del rango 45 - 54 años presentan un riesgo moderado y el $46,7 \%$ correspondientes a 28 pacientes del grupo $<45$ años presentan un riesgo bajo de desarrollar DM2.

Tabla 2. Relación entre la edad y el nivel de riesgo de DM2 en los pacientes afectados de tuberculosis.

\begin{tabular}{|c|c|c|c|c|c|c|c|c|c|c|c|c|}
\hline $\begin{array}{c}\text { Nivel de } \\
\text { riesgos de }\end{array}$ & \multicolumn{2}{|c|}{ Bajo } & \multicolumn{2}{|c|}{ Moderado } & \multicolumn{2}{|c|}{$\begin{array}{l}\text { Ligeramente } \\
\text { elevado }\end{array}$} & \multicolumn{2}{|c|}{ Alto } & \multicolumn{2}{|c|}{ Muy alto } & \multicolumn{2}{|c|}{ Total } \\
\hline Edad & $N^{\circ}$ & $\%$ & $N^{\circ}$ & $\%$ & $N^{\circ}$ & $\%$ & $N^{\circ}$ & $\%$ & $N^{\circ}$ & $\%$ & $N^{\circ}$ & $\%$ \\
\hline$<45$ años & 27 & 45 & 1 & 1,7 & 11 & 18,3 & 1 & 1,6 & 0 & 0 & 40 & 66,6 \\
\hline 45 - 54 años & 1 & 1,7 & 5 & 8,3 & 2 & 3,3 & 2 & 3,3 & 0 & 0 & 10 & 16,7 \\
\hline 55 - 64 años & 0 & 0 & 0 & 0 & 4 & 6,7 & 1 & 1,7 & 0 & 0 & 5 & 8,4 \\
\hline > 64 años & 1 & 1,7 & 0 & 0 & 2 & 3,3 & 1 & 1,7 & 1 & 1,7 & 5 & 8,4 \\
\hline
\end{tabular}

El gráfico 1 representa los porcentajes de los niveles de riesgo de desarrollar DM2 de los pacientes involucrados en el estudio; el $48 \%$ que representa la mayoría de los pacientes tienen un nivel de riesgo bajo, seguido por el $32 \%$ con un riesgo ligeramente elevado, el $10 \%$ con un riesgo moderado, el $8 \%$ tiene presenta un riesgo alto y solo el $2 \%$ presenta un nivel de riesgo muy alto de padecer DM2. 
Gráfico 1. Niveles de riesgo de desarrollar Diabetes Mellitus tipo 2 en los pacientes afectados de tuberculosis.

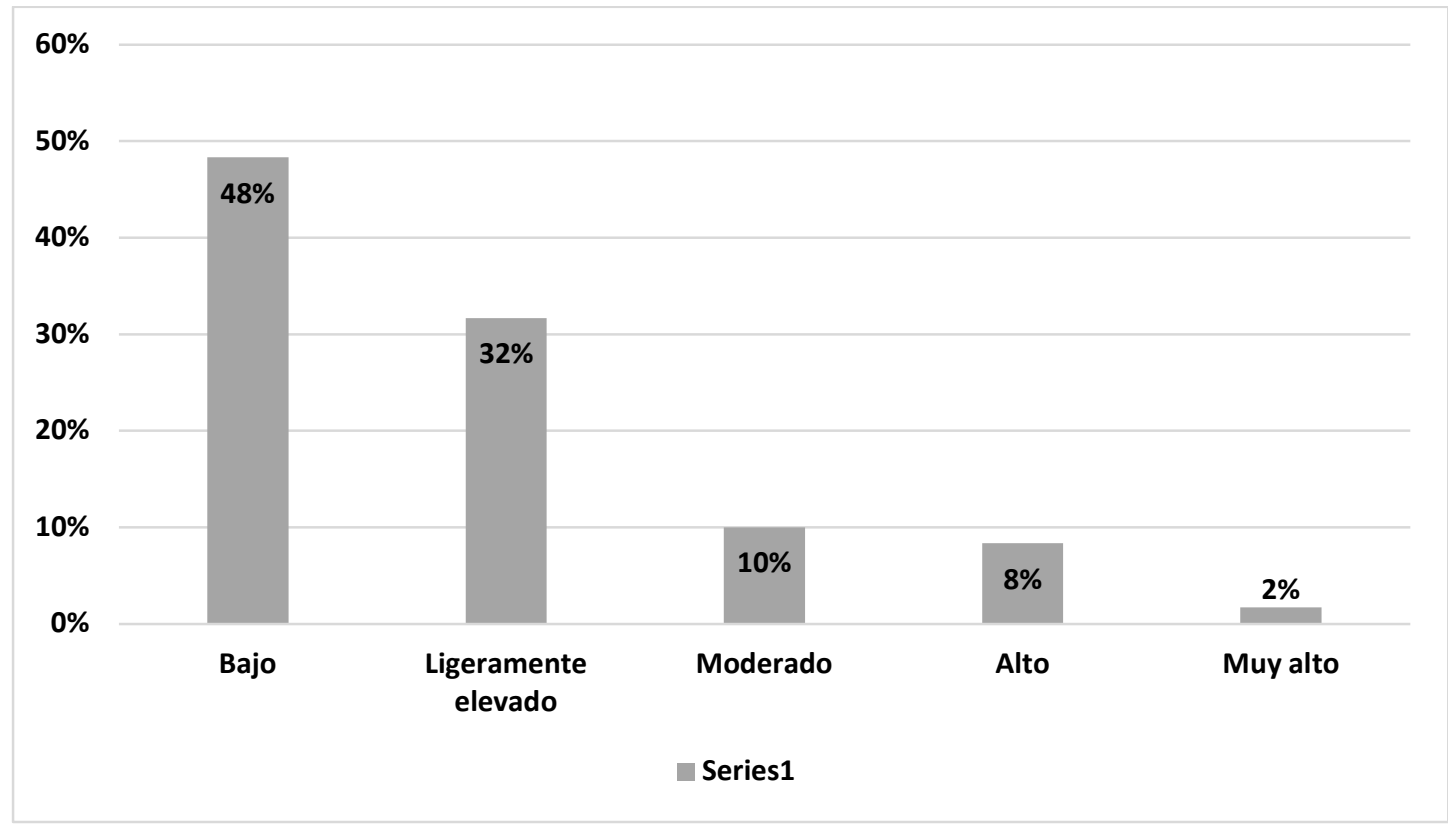

\section{DISCUSIÓN}

La diabetes es un problema de salud pública importante y una de las cuatro enfermedades no transmisibles (ENT) cuya carga los líderes mundiales se proponen aliviar mediante diversas medidas. Tanto el número de casos como la prevalencia de diabetes han aumentado progresivamente en los últimos decenios. A escala mundial se calcula que 422 millones de adultos tenían diabetes en 2014, por comparación con 108 millones en $1980{ }^{(16)}$.

Los factores de riesgo para desarrollar diabetes son numerosos según la literatura científica y estos a su vez son modificables y no modificables en relación con el individuo, en este estudio se evaluó el riesgo de desarrollar diabetes mellitus tipo 2 en los pacientes afectados con tuberculosis que se encuentran recibiendo tratamiento antifímico debido a su asociación descrita en diferentes investigaciones. La mayoría de los estudios publicados respecto a la asociación entre diabetes y tuberculosis parten de la premisa de que "padecer diabetes es un factor de riesgo para desarrollar tuberculosis", de ahí la importancia del artículo de Méndez et al (17), donde reporta que la prevalencia de tuberculosis latente (TBL) en pacientes con diabetes mellitus es de $35 \%$ y la diabetes se ha asociado con un incremento del riesgo de TBL ${ }^{(18)}$; en este estudio se analizó la dirección contraria de esta asociación, es decir el riesgo de desarrollar DM en los pacientes afectados con TB.

Luego de consultar varias bases de datos a nivel local, regional y mundial encontramos que actualmente no hay estudios similares que detallen la existencia de este riesgo, sin embargo, hay numerosos estudios que detallan el riesgo de TB en pacientes con DM por diferentes mecanismos.

En nuestro estudio encontramos que el sexo masculino presenta un mayor riesgo de desarrollar DM2 en condición de su enfermedad, contradiciendo lo que señalan varios estudios llevados a cabo en diferentes países donde se observan tasas de DM2 
superiores en mujeres que en hombres $(19,20)$ pudiendo atribuir esta contradicción al mayor riesgo que tienen los hombres de desarrollar tuberculosis como lo señala la OMS (1) "la tuberculosis mata a más de 3 millones de personas al año, en su mayoría hombres" ; con respecto a la edad tenemos que los grupos etarios <45 años y $45-54$ años en su totalidad son quienes presentan un mayor nivel de riesgo de desarrollar DM2 coincidiendo con lo reportado en otras investigaciones donde señalan que el riesgo aumenta con la edad y que los individuos entre 46 a 60 años la presentan mayor frecuencia(21,22); Jiménez Mejías, et al ${ }^{(23)}$., señala que la prevalencia de la DM tipo 2 (que representa en torno al $90 \%$ los casos de DM), está fuertemente asociada a la edad. Los datos de este estudio en forma general se concentraron en el nivel de riesgo bajo de desarrollar DM2, seguido por el nivel ligeramente moderado.

Si bien es cierto, estos datos pueden mirarse e interpretarse desde dos dimensiones diferentes, la primera es que los resultados sobre el nivel de riesgo estén modificados y asociados a la condición de padecer tuberculosis y estar recibiendo el tratamiento antifímico, o bien, la segunda opción pudiera ser que el riesgo de desarrollar DM2 ya estaba presente en este grupo de pacientes antes de que fueran afectados por TB, aunque esta sea solo una conjetura interpretativa, es importante que se realicen más estudios a este grupo vulnerable para poder fundamentar este sustento en la ciencia, de allí la relevancia del estudio.

\section{CONCLUSIÓN}

La población de pacientes afectados con tuberculosis, aunque presentan niveles de riesgo bajo, este se encuentra presente, por lo que resulta imprescindible que los profesionales encargados del manejo terapéutico de los pacientes con tuberculosis a nivel de la atención primaria tomen medidas encaminadas a la mitigación y control de este riesgo, educando a los pacientes sobre estilos de vida adecuados a su condición y así evitar la aparición de comorbilidades que afecten la adherencia terapéutica al tratamiento antifímico y el control de la enfermedad.

Se necesitan más estudios para poder determinar el riesgo de desarrollar Diabetes mellitus tipo 2 de forma real en los pacientes afectados con tuberculosis.

\section{REFERENCIAS}

1. Organización Mundial de la Salud. Tuberculosis [Internet]. World Health Organization. 2018 [citado 5 de septiembre de 2018]. Disponible en: http://www.who.int/es/news-room/fact-sheets/detail/tuberculosis

2. Organización Mundial de la Salud. Informe mundial sobre la tuberculosis 2016 [Internet]. World Health Organization. [citado 5 de septiembre de 2018]. Disponible en: http://apps.who.int/medicinedocs/en/d/Js23098en/

3. Censos IN de E y. Estadísticas de Nacimientos y Defunciones 2015 [Internet]. Instituto Nacional de Estadística y Censos. [citado 5 de septiembre de 2018]. Disponible en: http://www.ecuadorencifras.gob.ec/estadisticas-de-nacimientos-ydefunciones-2015/

4. González Hernández Y, Sada Díaz E, Escobar Gutiérrez A, Muños Torrico M, Torres Rojas M. Asociación de tuberculosis y diabetes mellitus: Mecanismos inmunológicos involucrados en la susceptibilidad. Rev Inst Nal Enf Resp Mex. 2009;22(1):8. 
5. Vidal P, https://www.facebook.com/pahowho. PAHO/WHO | The Dual Threat of Diabetes and Tuberculosis in the Americas [Internet]. Pan American Health Organization / World Health Organization. 2013 [citado 5 de septiembre de 2018]. Disponible

http://www.paho.org/hq/index.php?option=com_content\&view=article\&id=9112:2013the-dual-threat-diabetes-tuberculosis-americas\&ltemid=39447\&limitstart=2\&lang=en 6. Shewade HD, Jeyashree K, Mahajan P, Shah AN, Kirubakaran R, Rao R, et al. Effect of glycemic control and type of diabetes treatment on unsuccessful TB treatment outcomes among people with TB-Diabetes: A systematic review. PLoS ONE [Internet]. 23 de octubre de 2017 [citado 5 de septiembre de 2018];12(10). Disponible en: https://www.ncbi.nlm.nih.gov/pmc/articles/PMC5653348/

7. Gopathi N, Mandava V, Makala S. Tuberculosis and diabetes: the deadly duo. Int J Adv Med. 2015;241-5.

8. Jeon CY, Murray MB. Diabetes Mellitus Increases the Risk of Active Tuberculosis: A Systematic Review of 13 Observational Studies. PLoS Med [Internet]. julio de 2008 [citado 5 de septiembre de 2018];5(7). Disponible en: https://www.ncbi.nlm.nih.gov/pmc/articles/PMC2459204/

9. Ahmed M, Omer I, Osman SA, Ahmed-Abakur E. Association between pulmonary tuberculosis and Type 2 diabetes in Sudanese patients. The International Journal of Mycobacteriology. 2017;6(1):97.

10. Muñoz-Torrico M, Caminero-Luna J, Migliori GB, D'Ambrosio L, Carrillo-Alduenda $\mathrm{JL}$, Villareal-Velarde $\mathrm{H}$, et al. La diabetes se asocia con reacciones adversas graves en la tuberculosis multirresistente. Arch Bronconeumol. mayo de 2017;53(5):245-50.

11. Lawson L, Muc M, Oladimeji O, Iweha C, Opoola B, Abdurhaman ST, et al. Tuberculosis and diabetes in Nigerian patients with and without HIV. Int J Infect Dis. 1 de agosto de 2017;61:121-5.

12. Dave $P$, Shah A, Chauhan M, Kumar AMV, Harries AD, Malhotra S, et al. Screening patients with tuberculosis for diabetes mellitus in Gujarat, India. Public Health Action. 4 de noviembre de 2013;3(Suppl 1):S29-33.

13. Ministerio de Salud Publica del Ecuador. Prevención, diagnóstico, tratamiento y control de la tuberculosis. Guía de Práctica Clínica [Internet]. Dirección Nacional de Normatización; 2018. Disponible en: https://www.salud.gob.ec/wpcontent/uploads/2018/03/GP_Tuberculosis-1.pdf

14. Rydén L, Grant PJ, Ankēer SD, Berne C, Cosentino F, Danchin N, et al. Guía de práctica clínica de la ESC sobre diabetes, prediabetes y enfermedad cardiovascular, en colaboración con la European Association for the Study of Diabetes. Rev Esp Cardiol. febrero de 2014;67(2):136.e1-136.e56.

15. Avilés AGP, Rosas ERP, Bellot FT, Giles OA, Mendoza RD. Cuestionario FINDRISC FINnish Diabetes Risk Score para la detección de diabetes no diagnosticada y prediabetes. 2018;9.

16. Organización Mundial de la Salud. Informe mundial sobre la Diabetes [Internet]. Organización Mundial de la Salud; 2016. Disponible en: http://apps.who.int/iris/bitstream/handle/10665/254649/9789243565255-

spa.pdf?sequence=1

17. Méndez J, Sánchez É, Saavedra A, García-Herreros P. Prevalence of latent tuberculosis in patients with diabetes mellitus at a hospital in the city of Bogotá, Colombia. Acta Médica Colombiana. septiembre de 2017;42(3):8.

18. Bastidas AR. Búsqueda de tuberculosis latente en poblaciones especiales: Diabetes. Acta Médica Colombiana,. septiembre de 2017;42(3):3.

19. Sandín M, Espelt A, Escolar-Pujolar A, Arriola L, Larrañaga I. Desigualdades de género y diabetes mellitus tipo 2: La importancia de la diferencia. Av En Diabetol. 1 de mayo de 2011;27(3):78-87. 
20. Tang $M$, Chen $Y$, Krewski D. Gender-related differences in the association between socioeconomic status and self-reported diabetes. Int J Epidemiol. junio de 2003;32(3):381-5.

21. Cordero LCA, Rojas J, Bermúdez V. Prevalencia de la diabetes mellitus tipo 2 y sus factores de riesgo en individuos adultos de la ciudad de Cuenca- Ecuador. Avances en Biomedicina. abril de 2017;16(1):13.

22. Alcocer BS, Valle ARC, Ceh JGG. Identificación de Factores de Riesgo de la Diabetes Mellitus Tipo 2 en Adultos de 30 a 60 años de edad en la Comunidad de Isla Aguada, Municipio de Ciudad del Carmen, Campeche. RIDE Revista Iberoamericana para la Investigación y el Desarrollo Educativo [Internet]. junio de 2016 [citado 2 de octubre de 2018];6(12). Disponible en: http://www.redalyc.org/articulo.oa?id=498153966027

23. Martínez Ruiz V. Efecto De La Edad Sobre La Evolución De La Prevalencia De Diabetes. Nutr Hosp. 1 de junio de 2014;29(6):1335-1338. 